\title{
Differential expression of major histocompatibility complex class I in developmental glioneuronal lesions
}

Avanita S Prabowo ${ }^{1}$, Anand M lyer ${ }^{1}$, Jasper J Anink ${ }^{1}$ Wim GM Spliet ${ }^{2}$, Peter C van Rijen ${ }^{2}$ and Eleonora Aronica ${ }^{1,3,4^{*}}$

\begin{abstract}
Purpose: The expression of the major histocompatibility complex class I (MHC-I) in the brain has received considerable interest not only because of its fundamental role in the immune system, but also for its non-immune functions in the context of activity-dependent brain development and plasticity.

Methods: In the present study we evaluated the expression and cellular pattern of MHC-I in focal glioneuronal lesions associated with intractable epilepsy. MHC-I expression was studied in epilepsy surgery cases with focal cortical dysplasia (FCD I, $n=6$; FCD Ila, $n=6$ and FCD IIb, $n=15$ ), tuberous sclerosis complex (TSC, cortical tubers; $n=6$ ) or ganglioglioma (GG; $n=15)$ using immunocytochemistry. Evaluation of T lymphocytes with granzyme-B ${ }^{+}$ granules and albumin immunoreactivity was also performed.

Results: All lesions were characterized by MHC-I expression in blood vessels. Expression in both endothelial and microglial cells as well as in neurons (dysmorphic/dysplastic neurons) was observed in FCD II, TSC and GG cases. We observed perivascular and parenchymal $T$ lymphocytes (CD8 ${ }^{+}$, T-cytotoxic) with granzyme- ${ }^{+}$granules in FCD IIb and TSC specimens. Albumin extravasation, with uptake in astrocytes, was observed in FCD Ilb and GG cases.

Conclusions: Our findings indicate a prominent upregulation of $\mathrm{MHC}-\mathrm{I}$ as part of the immune response occurring in epileptogenic glioneuronal lesions. In particular, the induction of $\mathrm{MHC}-1$ in neuronal cells appears to be a feature of type II FCD, TSC and GG and may represent an important accompanying event of the immune response, associated with blood-brain barrier dysfunction, in these developmental lesions.
\end{abstract}

Keywords: Focal cortical dysplasia, Ganglioglioma, Cortical tubers, Neurons, Microglia, Major histocompatibility complex $(\mathrm{MHC})$ class I

\section{Introduction}

Recent clinical and neuropathological evidence supports the critical role of a sustained inflammatory reaction in glioneuronal lesions with activation of both the innate and the adaptive immune response and involvement of different inflammatory pathways (for reviews see [1-4]). Interestingly, proinflammatory molecules have been shown to alter neuronal excitability and, in experimental models, to decrease the seizure threshold, contributing to neuronal cell death [2-4].

\footnotetext{
*Correspondence: e.aronica@amc.uva.nl

'Department of (Neuro)Pathology, Academic Medical Center, University of

Amsterdam, Meibergdreef 9, Amsterdam 1105 AZ, The Netherlands

${ }^{3}$ SEIN - Stichting Epilepsie Instellingen Nederland, Heemstede, The Netherlands

Full list of author information is available at the end of the article
}

According to the current histopathological classification system [5], focal cortical dysplasia (FCD) has been classified into type I, characterized by cortical dyslamination (with three FCD subtypes according to the pattern of dyslamination), and type II, characterized by additional cytological abnormalities (FCD IIa with dysmorphic neurons and FCD IIb with dysmorphic neurons and balloon cells).

In a recent study [6], we confirmed the occurrence of complex inflammatory changes (involving both glial and neuronal cells) in FCD specimens and demonstrated that the severity of these changes is greater in FCD IIb than in specimens from patients with FCD I. The activation of components of the adaptive immunity, with the presence of $\mathrm{T}$ lymphocytes $\left(\mathrm{CD}^{+}\right.$, $\mathrm{T}$-cytotoxic/suppressor 
immunophenotype), has also been mainly observed in FCD IIb specimens [6].

Whether these inflammatory changes represent a feature common to different developmental glioneuronal lesions and whether induction of major histocompatibility complex (MHC) class I molecules may be involved still needs to be clarified.

MHC-I molecules play a fundamental role in the immune system, in particular in the context of the adaptive immune response, but have also been shown to have non-immune functions, being involved in the regulation of activity-dependent brain development and plasticity (for a review see [7]). Interestingly, glial and neuronal MHC-I upregulation has been observed in brain specimens from patients with Rasmussen's encephalitis (a severe inflammatory epileptic encephalopathy of childhood) and intractable epilepsy and it has been suggested that it plays a critical role in antigen-specific cytotoxicity $[8,9]$.

The aim of the present study was to determine whether MHC-I is induced in focal glioneuronal lesions associated with intractable epilepsy. We evaluated the cellular distribution of MHC-I within a large spectrum of glioneuronal lesions, including focal cortical dysplasia type I, type IIa and type IIb, cortical tubers from patients with tuberous sclerosis complex (TSC) and gangliogliomas (GGs). Additionally, the presence of $\mathrm{T}$ lymphocytes with granzyme- $\mathrm{B}^{+}$granules and albumin immunoreactivity within the lesion and its uptake into astrocytes were also analyzed.

\section{Material and methods}

\section{Patients and controls}

We examined a total of 48 surgical specimens: 6 FCD I (3, Ia; 3, Ib), 6 FCD IIa, 15 FCD IIb, 6 cortical tubers from patients with TSC and 15 GG. The cases included in this study were obtained from the departments of neuropathology of the Academic Medical Center (University of Amsterdam, UvA) in Amsterdam and the University Medical Center in Utrecht (UMCU), the Netherlands. The clinical characteristics derived from the patients' medical records are summarized in Table 1 . Patients underwent therapeutic surgical resection for refractory epilepsy and had, predominantly, medically intractable complex partial seizures. The postoperative seizure outcome was classified according to Engel [10]. All the patients included in our series did not have any apparent seizure activity in the $24 \mathrm{~h}$ before surgery. Patients who underwent implantation of strip and/or grid electrodes for chronic subdural invasive monitoring before resection were excluded from this study.

To grade the degree of FCD, we followed the international consensus classification recently proposed [5]. All patients with cortical tubers fulfilled the diagnostic criteria for TSC [11]. For GG, we used the revised WHO classification of tumors of the central nervous system [12]. In five patients (one FCD, one TSC and three GG) a significant amount of perilesional tissue (normalappearing cortex/white matter adjacent to the lesion) was resected as well. Brain tissue from patients with viral encephalitis (rabies encephalitis [13]; herpes simplex encephalitis (female, age at autopsy: 69 years) and Rasmussen's encephalitis $(n=6 ; 4$ females and 2 males; mean age at surgery: 20.6 years, range: 16 to 26 ) were also examined as positive controls. In addition, normalappearing control cortex/white matter was obtained at autopsy from six young adult control patients (Table 1), without a history of seizures or other neurological diseases. All autopsies were performed within $12 \mathrm{~h}$ after death. Informed consent was obtained for the use of brain tissue. Tissue was obtained and used in a manner compliant with the Declaration of Helsinki.

Table 1 Summary of clinical findings for epilepsy patients and controls

\begin{tabular}{|c|c|c|c|c|c|}
\hline Pathology type & $\begin{array}{l}\text { Number of } \\
\text { cases }\end{array}$ & $\begin{array}{l}\text { Gender } \\
\text { (males/females) }\end{array}$ & $\begin{array}{l}\text { Mean age at surgery } \\
\text { (years/range) }\end{array}$ & Localization & $\begin{array}{l}\text { Mean duration of } \\
\text { epilepsy (years/range) }\end{array}$ \\
\hline \multirow[t]{2}{*}{$\overline{F C D ~ I ~}$} & 6 & $3 / 3$ & 27.8 (19 to 39 ) & 4 Temporal & 7.7 (6 to 13$)$ \\
\hline & & & & 2 Frontal & \\
\hline \multirow[t]{2}{*}{ FCD lla } & 6 & $3 / 3$ & 24.3 (11 to 24$)$ & 3 Frontal & 6.1 (2 to 11$)$ \\
\hline & & & & 3 Temporal & \\
\hline \multirow[t]{2}{*}{ FCD $\| b$} & 15 & $7 / 8$ & 23.2 (17 to 41$)$ & 8 Temporal & 7.8 (3 to 15$)$ \\
\hline & & & & 7 Frontal & \\
\hline \multirow[t]{3}{*}{ Cortical tubers (TSC) } & 6 & $3 / 3$ & 17.8 (5 to 35 ) & 3 Frontal & 13.5 (2.8 to 34$)$ \\
\hline & & & & 3 Temporal & \\
\hline & & & & 2 Parietal & \\
\hline GG & 15 & $8 / 7$ & 29.2 (15 to 46$)$ & Temporal & 7.8 (3 to 15$)$ \\
\hline Controls (no epilepsy) & 6 & $3 / 3$ & 30.1 (20 to 41$)$ & Temporal & - \\
\hline
\end{tabular}

FCD, focal cortical dysplasia; TSC, tuberous sclerosis complex; GG, ganglioglioma. 


\section{Tissue preparation}

Formalin-fixed, paraffin-embedded tissue samples (one representative paraffin block per case containing the complete lesion or the largest part of the lesion resected at surgery) were sectioned at $6 \mu \mathrm{m}$ and mounted on precoated glass slides (Star Frost, Waldemar Knittel GmbH, Braunschweig, Germany). Sections of all specimens were processed for hematoxylin eosin, luxol fast blue and Nissl stains as well as for immunocytochemical stainings for a number of neuronal and glial markers, which are described below.

\section{Immunocytochemistry}

The primary antibodies used in the study are summarized in Table 2. Single-label immunocytochemistry was developed using the Powervision kit (Immunologic, Duiven, the Netherlands). 3,3-diaminobenzidine (Sigma, St Louis, USA) was used as the chromogen. Sections were counterstained with hematoxylin.

For double-labeling of MHC-I with GFAP, MAP-2 or pS6 (as well as of albumin with CD31 or GFAP and GrB with GFAP) sections were, after incubation with the primary antibodies overnight at $4^{\circ} \mathrm{C}$, incubated for $2 \mathrm{~h}$ at room temperature with Alexa Fluor ${ }^{\circledR}$ 568-conjugated anti-rabbit and Alexa Fluor ${ }^{\circledR} 488$ anti-mouse immunoglobulin G (IgG) or anti-goat IgG (Molecular Probes, The Netherlands; 1:100). Sections were then analyzed using a laser scanning confocal microscope (Leica TCS Sp2, Wetzlar, Germany).

For double-labeling of MHC-I with NeuN, sections were incubated with the first primary antibody. AntiMHC-I was visualized with a polymer-alkaline phosphatase (AP)-labeled anti-rabbit antibody (BrightVision \#DPVM55AP, Immunologic, Duiven, The Netherlands) and Vector Red (AP substrate kit III, \#SK-5100, Vector labs, Burlingame, CA, USA) as the chromogen. To remove the first primary antibody (MHC-I), the sections were incubated at $121^{\circ} \mathrm{C}$ in a citrate buffer $(0.01 \mathrm{M}$, $\mathrm{pH}$ 6.0) for $10 \mathrm{~min}$. Sections were then incubated for $1 \mathrm{~h}$ at room temperature with the second primary antibody (NeuN). The second primary antibodies were visualized with poly-AP anti-rabbit antibody (BrightVision \#DPVM55AP, Immunologic, Duiven, The Netherlands) and Vector Blue (AP substrate kit III, \#SK-5300, Vector labs, Burlingame, CA, USA) as the chromogen.

\section{Evaluation of immunostaining}

All labeled tissue sections were evaluated by two independent observers blind to clinical data, for the presence or absence of various histopathological parameters and specific immunoreactivity for the different markers. Two representative sections per case were stained and assessed for the MHC-I and albumin. The intensity of the staining was evaluated as previously described $[6,14]$, using a semi-quantitative scale ranging from 0 to 3 (0: negative; 1 : weak; 2 : moderate; 3 : strong immunoreactivity). All areas of the specimen were examined and the score represents the predominant cell staining intensity found in each case. The approximate proportion of cells (microglia, neurons and endothelial cells/blood vessels) showing MHC-I immunoreactivity (1: single to $10 \%$; 2 : $11 \%$ to $50 \%$; 3 : $>50 \%$ ) was also scored to give information about the relative number ('frequency' score) of positive cells within the specimens with a malformation of cortical development. In the case of a disagreement, independent reevaluation was performed by both observers to define the final score. As proposed before $[6,15]$, the product of these two values (intensity and frequency scores) was taken to give the overall score

Table 2 Immunocytochemistry: primary antibodies

\begin{tabular}{|c|c|c|c|}
\hline Antigen & Primary antibody & Source & Dilution \\
\hline Glial fibrillary acidic protein (GFAP) & Polyclonal rabbit & DAKO, Glostrup, Denmark & $1: 4,000$ \\
\hline Neuronal nuclear protein (NeuN) & Mouse clone MAB377 & Chemicon, Temecula, CA, USA & $1: 2,000$ \\
\hline HLA-DP, DQ, DR (MHC-II) & Mouse clone CR3/43 & DAKO, Glostrup, Denmark & $1: 1000$ \\
\hline HLA-A, B, C (MHC-I) & Mouse clone $\mathrm{HC}-10$ & Gift from Prof. J. Neefjes, NKI, the Netherlands & $1: 200$ \\
\hline Vimentin & Mouse clone V9 & DAKO, Glostrup, Denmark & $1: 400$ \\
\hline Neurofilament (NF) & Mouse clone SMI311 & Sternberger Monoclonals, Lutherville, MD, USA & $1: 1,000$ \\
\hline Microtubule associated protein (MAP2) & Mouse clone HM2 & Sigma,St. Louis, IL, USA & $1: 100$ \\
\hline Microtubule associated protein (MAP2) & Polyclonal rabbit & Millipore, Billerica, MA, USA & $1: 500$ \\
\hline Phospho-S6 ribosomal protein (ps6) & Polyclonal rabbit (Ser235/236; pS6) & Cell Signaling Technology, Beverly, MA, USA; & $1: 50$ \\
\hline CD68 & Mouse clone PG-M1 & DAKO, Glostrup, Denmark & $1: 200$ \\
\hline CD31 & Mouse clone JC70A & DAKO, Glostrup, Denmark & $1: 10$ \\
\hline Albumin & Rabbit polyclonal & DAKO, Glostrup, Denmark & $1: 20,000$ \\
\hline Granzyme B (GrB) & Mouse clone GrB-7 & Monosan, Uden, The Netherlands & $1: 100$ \\
\hline
\end{tabular}




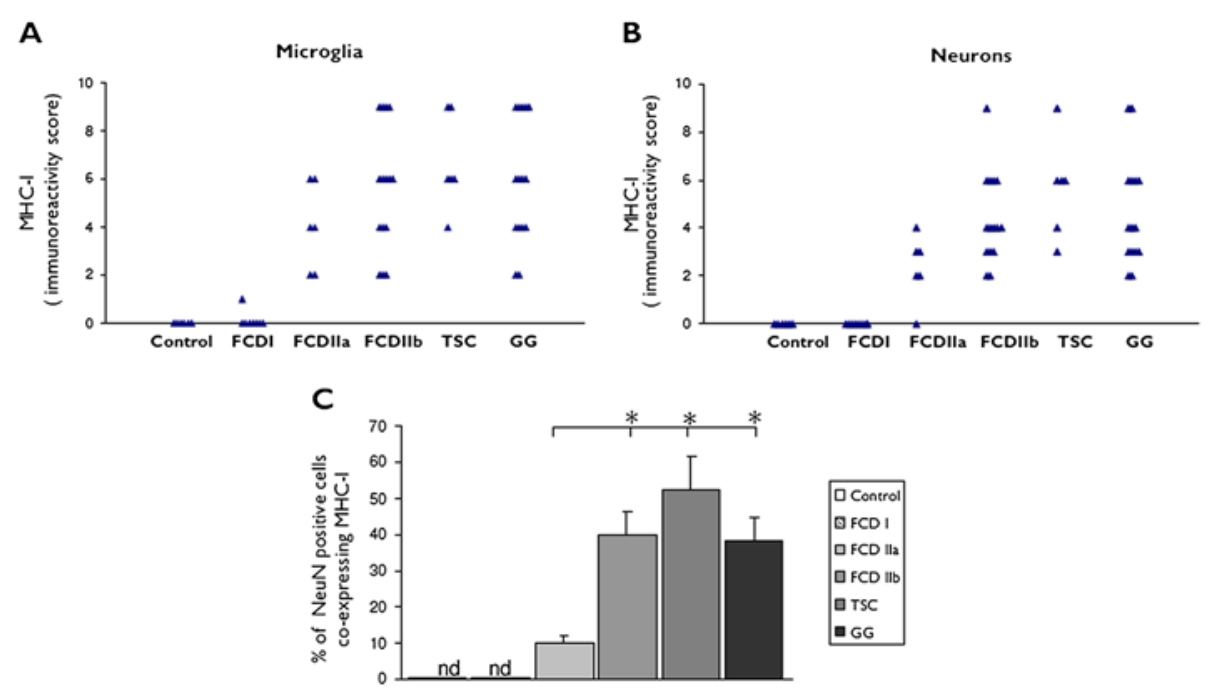

Figure 1 Evaluation of MHC-I expression in control and FCD I, FCD Ila, FCD Ilb, TSC and GG. (A) and (B): MHC-I immunoreactivity scores (total score; see material and methods for details) in microglial cells and neurons. (C): Graph showing percentages of neuronal cells immunoreactive for NeuN and co-expressing MHC-I. Data are expressed as mean \pm standard error of mean (SEM). P $<0.05$ compared to FCD I and controls. FCD, focal cortical dysplasia; TSC, tuberous sclerosis complex; GG, ganglioglioma; MHC-I, major histocompatibility complex class l; ND, not determined; nd, not detectable.

(immunoreactivity total score), which is shown in Figure 1 (MHC-I) and Figure 2 (albumin). Neuronal cell bodies were differentiated from glia and glia-neuronal balloon cells or giant cells based on morphology.

To analyze the percentage of double-labeled cells positive for MHC-I and NeuN, digital images of eight representative fields per section (magnification 20 times) were collected (Leica DM5000B). Images were analyzed with a Nuance VIS-FL Multi-spectral Imaging System (Cambridge Research Instrumentation; Woburn, MA) as previously described $[16,17]$. The total number of cells stained with MHC-I or NeuN, as well as the number of cells double labeled with both were counted visually and percentages were calculated (expressed as mean \pm SEM) of cells co-expressing NeuN and MHC-I.

Quantitative analysis was performed for $\mathrm{GrB}$ and the numbers of positive cells were quantified as previously described $[15,18]$. Quantitative analysis of the staining intensity in endothelial cells was also performed. The relative optical density ratio (ODR) of endothelial cells immunolabeled with MHC-I was calculated as previously described [19]. The degree of MHC-I expression in microvessels was evaluated by counting the numbers of vessels expressing the protein in two non-overlapping microscopic fields (field size $1 \mathrm{~mm}^{2}$ ) of control and FCD IIb specimens ( $n=6$ in each group). Results were expressed as a normalized mean \pm SEM of MHC-I positive vessels per microscopic field, taking into account the total number of microvessels in control and FCD specimens, assessed by counting the number of CD31positive vessels in adjacent serial sections, as previously described [20].

\section{Statistical analysis}

Statistical analyses were performed with SPSS for Windows (SPSS 11.5, SPSS Inc., Chicago, IL, USA). The
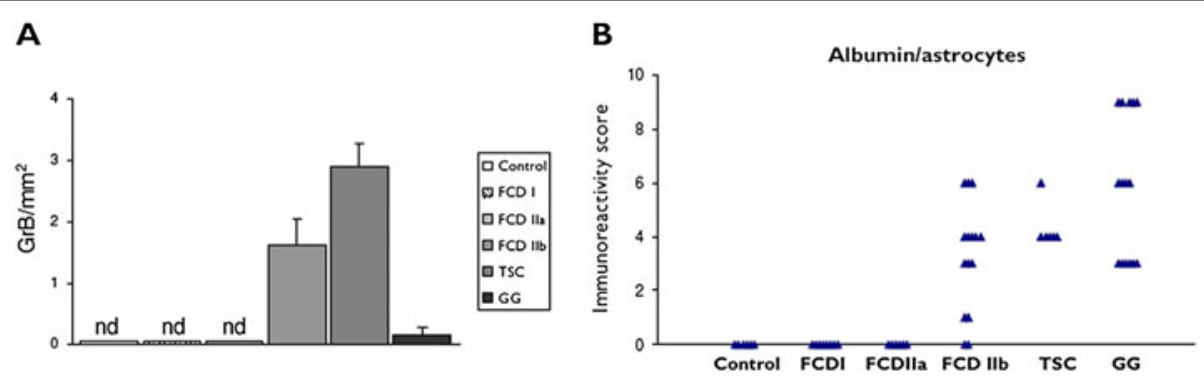

Figure 2 Evaluation of GrB positive cells and albumin immunoreactivity (IR) in astrocytes. (A): Graph showing GrB cell counting in control cortex, FCD I, FCD Ila, FCD Ilb and GG. * $P<0.05$, compared to GG, FCD I, FCD lla and controls. (B): Albumin immunoreactivity scores (total score; see material and methods for details) in astrocytes. 
two-tailed Student's t-test was used to assess differences between groups. To assess differences between more than two groups a non-parametric Kruskal-Wallis test followed by a Mann-Whitney U test were used. Correlation between immunostaining (number of positive cells) and different clinical variables (duration of epilepsy, seizure frequency, age at surgery, age at seizure onset and epilepsy outcome) were assessed using the Spearman's rank correlation test. A value of $P<0.05$ was defined as being statistically significant.

\section{Results}

\section{Case material and histological features}

The clinical features of the cases included in this study are summarized in Table 1. All patients had a history of chronic pharmaco-resistant epilepsy. Age at surgery, seizure duration and seizure frequency were not statistically different between patients with FCD I, FCD II and GG in this cohort. Postoperatively, 67\% of patients in this cohort were completely seizure free. In this study, we excluded patients with a mild degree of cortical dysplasia (mild malformation of cortical development). The FCD cases included displayed the histopathological features of FCD Ib or FCD IIa and IIb, according to the international consensus classification [5].

The histopathological features of the cortical tuber samples included cortical dyslamination, giant cells, dysplastic neurons and astrogliosis $[21,22]$. TSC1 mutations were detected in one patient and TSC 2 mutations in five patients. In agreement with previous studies [6,23-25], the expression of pS6 (indicating the activation of the mTOR signal transduction pathway) was observed within all tubers, and FCD II and GG samples in our cohort. All of the 15 FCD IIb samples had a neuronal labeling index (frequency score) greater than $50 \%$. Of the 6 FCD IIa samples with pS6-positive neuronal cells, 3 (50\%) had a labeling index between $1 \%$ and $10 \%$, and the other $3(50 \%)$ had a labeling index between 11\% and $50 \%$. In contrast, pS6 protein expression was not detected in FCD I cases, as in the normal control specimens. The number of HLA-DR (MHC-II) immunoreactive cells was not significantly different between FCD IIa and IIb in our cohort, but was higher compared to FCD I, as previously reported [6].

\section{MHC-I expression in FCD I and II}

In the cortical autopsy of the human control, as well as in the normal-appearing cortex adjacent to the lesions (not shown), MHC-I was confined to blood vessels, and was not detected either in neurons or in glial cells in both cortex and white matter throughout all cortical layers (Figure 3A,B).

In agreement with previous observations, in both viral [13] and Rasmussen's encephalitis [8,9] immunoreactivity for MHC-I was also detected in lymphocytes, microglial cells, astrocytes and neurons (not shown).

In FCD I, MHC-I displayed an immunoreactivity pattern as in the controls, with expression in blood vessels, but no detectable immunoreactivity in glial or neuronal cells (Figure 3C and Figure 1A,B); in one case weak immunoreactivity was detected in a few microglial cells (Figure 1A).

In FCD IIa and IIb, consistent MHC-I expression was also observed in neurons and glial cells with the morphology of microglial cells (Figure 3D,E,F,G and Figure 1A, B). Double labeling confirmed MHC-I expression in cells of the microglial/macrophage lineage (HLA-DR positive cells; Figure 3 and inserts in $3 E$ and $3 G$ ), in neurons (MAP-2 and NeuN positive cells; not shown), but not in astrocytes (Figure 3; insert (b) in $3 \mathrm{E}$ ). The mean number of MHC-I positive neurons was found to be significantly higher in FCD IIb than in FCD IIa, whereas no significant differences were detected compared to TSC and GG specimens (Figure 1C). We did not detect MHC-I expression in balloon cells (Figure 3E,G). MHC-I immunoreactivity in microvessel endothelium was not significantly different compared to the control cortex (autopsy; Table 3, and/or the perilesional cortex, not shown). No significant correlation was found between the increased MHC-I staining in neurons in FCD II and the different clinical variables. However, a positive correlation was detected between the MHC-I staining in microglia and the duration of epilepsy (Spearman's rank correlation coefficient for FCD II, $r=0.865$ with $P<0.05$ ).

\section{MHC-I expression in cortical tubers (TSC)}

The expression pattern of MHC-I in cortical tubers of TSC patients resembled that observed in FCD II, with MHC-I positive blood vessels, neurons and microglial cells, but not astrocytes (Figure 3H,I and Figure 1A,B). The mean number of MHC-I positive neurons was found to be significantly higher in TSC than in FCD IIa, whereas no significant differences were detected compared to FCD IIb and GG specimens (Figure 1C). A large majority of giant cells were negative for MHC-I, only occasionally (in two cases) was MHC-I expression detected in sporadic giant cells within the white matter (Figure 3I). No significant correlation was found between the increased MHC-I staining in neurons in TSC and the different clinical variables. However, a positive correlation was detected between the MHC-I staining in microglia in TSC and the duration of epilepsy (Spearman's rank correlation coefficient for TSC, $r=0.835$ with $P<0.05$ ).

\section{MHC-I expression in GG}

In GG specimens, MHC-I immunoreactivity was detected in blood vessels, neurons and microglial cells, but 




Figure 3 Distribution of MHC-I immunoreactivity (IR) in FCD, TSC and GG. (A)-(B) Control cortex (A) and white matter (wm; B): MHC-I IR in blood vessels (arrows). (C) FCD I: MHC-I IR in blood vessels (arrows). (D) FCD lla: MHC-I IR in blood vessels (arrows), dysmorphic neurons (DN; asterisks) and microglial cells (arrowheads). Insert in (D) shows a neuron (asterisk) positive for MHC-I and both MHC-I and MHC-II IR in blood vessels (arrows). (E)-(G) FCD llb: strong MHC-I IR in cortex, (E) and (F) and wm, $(\mathbf{G})$ with expression in DN (arrowheads in $(\mathbf{E})$ and asterisk in $(\mathbf{F})$ ). MHC-I IR was also detected in blood vessels (arrows in (E)) and in microglial cells (insert (a) in (E), arrowheads in (F) and (G)). The balloon cells are negative (asterisks in $(\mathbf{G})$ ). Insert (b) in (E): absence of co-localization of MHC-I with GFAP in astrocytes. Insert (c) in (E) and the insert in (G): co-localization of MHC-I with MHC-II in microglial cells (the arrow in (c) indicates a microglial cell). (H), (I) TSC: strong MHC-I IR in both cortex $(\mathbf{H})$ and wm (I) with expression in DN (arrowheads in (H)), blood vessels (arrows in $(\mathbf{H})$ and $(\mathbf{I})$ ) and microglia (arrowhead in (I)). Giant cells are negative (asterisks). MHC-I IR is occasionally detected in giant cells (insert in (I)). Insert (a) in (H): co-localization with NeuN. Insert (b) in $(\mathbf{H})$ : co-localization with pS6. Insert (c) in (H): co-localization with MAP-2. (J) GG: MHC-I IR in dysplastic neurons (arrowheads; high magnification in insert (a)) and blood vessels (arrow). Insert (b) in (J): co-localization with NeuN. Insert (c) in (J): co-localization with MHC-II in microglial cells. Insert (d) in (J): absence of co-localization with GFAP. Scale bars: (A)-(C) and (E): $150 \mu \mathrm{m} ;(\mathbf{D}),(\mathbf{G})-(\mathbf{I}): 40 \mu \mathrm{m} ;(\mathbf{F}): 25 \mu \mathrm{m} ;(\mathbf{J}): 80 \mu \mathrm{m}$. 
not in tumor astrocytes (Figure 3J and Figure 1A,B). The mean number of MHC-I positive neurons was found to be significantly higher in GG than in FCD IIa, whereas no significant differences were detected compared to FCD IIb and TSC specimens (Figure 1C).

No significant correlation was found between the increased MHC-I staining in neurons in GG and the different clinical variables. However, a positive correlation was detected between the MHC-I staining in microglia and the duration of epilepsy (Spearman's rank correlation coefficient for GG, $r=0.847$ with $P<0.05$ ).

\section{T lymphocytes with granzyme $\mathrm{B}^{+}$granules}

In agreement with previous observations [6,26,27], inflammatory infiltrates in glioneuronal lesions, such as FCD IIb, contain $\mathrm{T}$ lymphocytes $\left(\mathrm{CD}^{+}, \mathrm{T}\right.$-cytotoxic immunophenotype) (Figure 4A).

In FCD IIb and TSC, we detected CD8 positive cells with granzyme- $\mathrm{B}^{+}$granules. These cells were often perivascularly located, but occasionally in the vicinity (or in close opposition) to dysmorphic neurons and astrocytes (Figure 4B,C,D,E and Figure 2A). Granzyme B immunoreactivity was not detected in FCD I or IIa, and only occasionally (one case) in GG specimens (Figure 2A).

\section{Albumin extravasation and immunoreactivity in astrocytes}

Alterations in blood-brain barrier permeability were detected using albumin immunocytochemistry in FCD IIb, TSC and GG specimens (Figure 4H,I,J,K,L and Figure 2B). In FCD IIb and TSC specimens, albumin immunoreactivity, with diffuse cytoplasmatic staining, was detected in perilesional astrocytes and in perivascularly located balloon/giant cells in FCD IIb and TSC specimens (Figure 4H,I,J,K); double labeling confirmed co-localization in GFAP positive cells (Figure 4J,K). Strong albumin immunoreactivity was observed within GG specimens in tumor astrocytes (Figure 4L and Figure 2B).

\section{Discussion}

In the present study we provide evidence of neuronal and microglial MHC-I expression in epileptogenic glioneuronal lesions. The neuronal expression of MHC-I

Table 3 MHC-I immunoreactivity in microvessel endothelium $^{a}$

\begin{tabular}{lcc}
\hline & Control $(\boldsymbol{n}=\mathbf{6})$ & FCD IIb $(\boldsymbol{n}=\mathbf{6})$ \\
\hline Intensity score & $1.47 \pm 0.06$ & $1.52 \pm 0.10$ \\
ODR & $2.8 \pm 0.3$ & $3.1 \pm 0.4$ \\
\hline
\end{tabular}

${ }^{a}$ Scoring of the histological specimens was performed as described in the Materials and methods section. Values represent the mean \pm SEM of the number of samples indicated in parentheses. ODR: relative optical density ratio of CD31-immunolabeled endothelium. was, however, only detected in FCD II, but not in FCD I specimens or in the perilesional region (despite the absence of significant differences in seizure frequency and duration). Interestingly, MHC-I expression in neurons was significantly higher in FCD IIb compared to FCD IIa and the large majority of balloon/giant cells did not express detectable levels of MHC-I. These observations confirm the difference between FCD I and II [6], suggest some differences between IIa and IIb and indicate that induction of MHC-I is not simply an effect of seizure activity.

Several studies demonstrate that expression of MHC-I can be upregulated in glia and neurons in response to different types of challenges, including injury, infections (chronic and acute), central administration of endotoxins and exposure to different cytokines ([28-33] reviewed in [7]). Cytokines have been show to differentially regulate MHC-I induction in neurons [31,34,35]. Previous studies have demonstrated prominent expression of components of the IL-1R/TLR signaling pathways in neuronal cells in epileptogenic glioneuronal lesions $[6,26,27,36]$. Signaling through these pathways leads to activation of the transcription factor, nuclear factor-kappa B (NF-kB) [37]. Interestingly, it has been suggested that activation of NF- $\kappa B$ plays a role in induction of MHC-I [38,39]. Thus, NF-kB-dependent mechanisms of regulation may contribute to the more prominent MHC-I expression detected in FCD II (associated with consistent activation of IL-1R/TLR signaling pathways [6]) compared to FCD I.

Endogenous peptides presented by MHC-I molecules (called MHC-I peptides (MIPs) or the immunopeptidome) represent the key to self/non-self-discrimination by cells of the immune system. In this respect, a recent study confirmed previous observations indicating that the immune system is tolerant to MIPs expressed at physiological levels but may promote immune responses towards self MIPs present in excessive amounts [40,41]. This may be important in the context, of the suggestion that neuronal MHC-I expression mediates removal of dysfunctional neurons [30]. The study by Caron et al. also suggests that changes in mTOR signaling can affect the expression of MHC-I and the repertoire of MIPs presented by MHC-I [41]. These observations highlight the complexity of MHC-I regulation and indicate the need for further analysis of the effect of mTOR modulation in lesions (such as FCD II, TSC and GG) in which this pathway is involved. Interestingly, a recent study suggests a novel viral etiology for FCD IIB [42], which could explain the constitutive activation of mTOR, as well as the induction of MHC-I, in this focal malformation of cortical development.

Interestingly, neuronal MHC-I expression has been reported in Rasmussen's encephalitis and it has been 


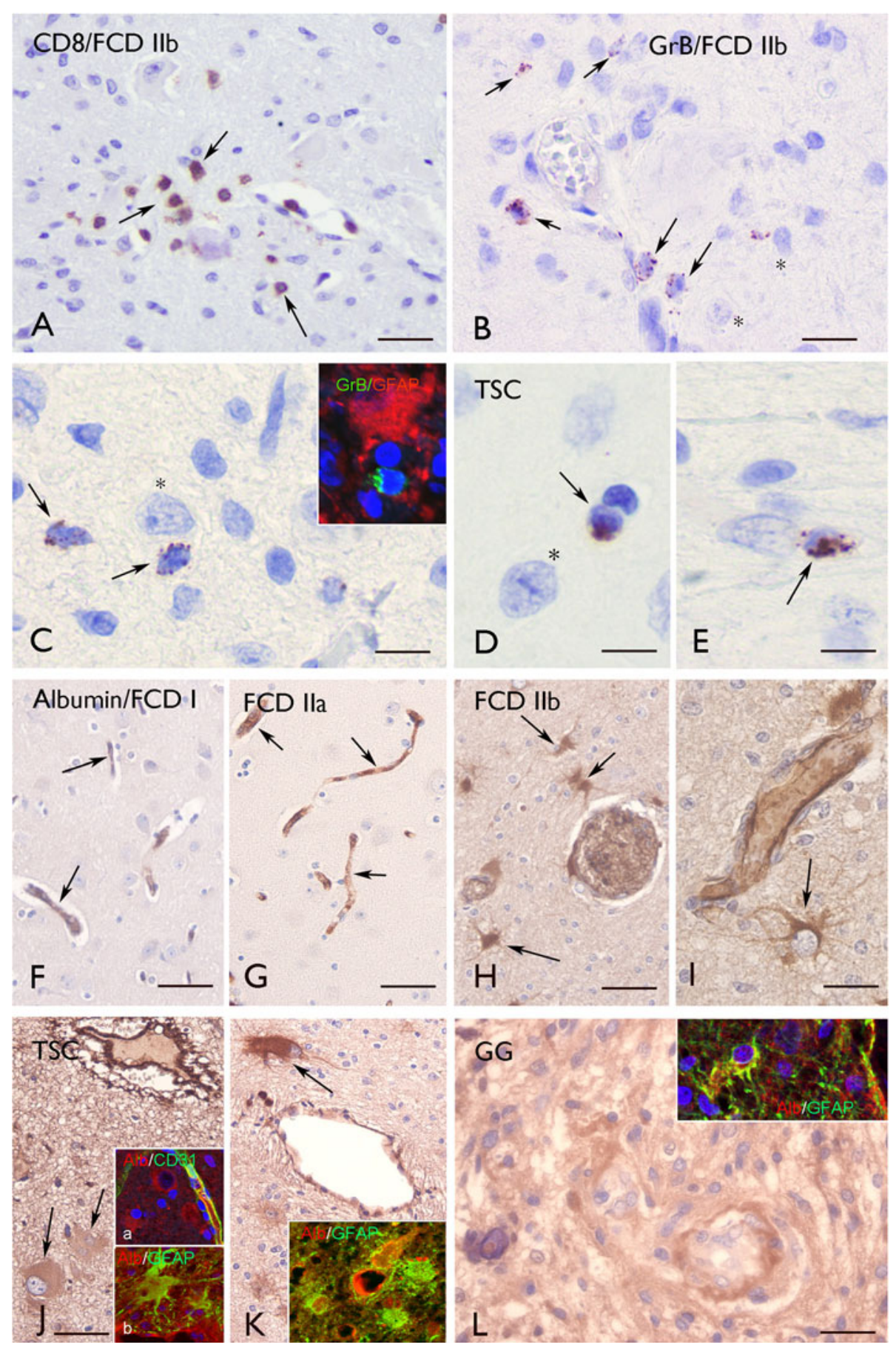

Figure 4 Granzyme B (GrB) and albumin immunoreactivity (IR). (A)-(E) Granzyme B (GrB) IR. (A) Perivascular CD8 positive cells (arrows) for FCD Ilb. (B) and (C) GrB positive cells (arrows) for FCD Ilb around blood vessels and in the vicinity of dysmorphic neurons (asterisks). Insert in (C) shows a GrB positive cell near an astrocyte (GFAP positive). (D) and (E) GrB positive cells in cortical tubers (arrows) for TSC, also the vicinity of a dysmorphic neuron (asterisk in (D)). (F)-(L) Albumin IR. For FCD I (F) and FCD Ila (G) there is an absence of glial IR. Albumin IR was detected in blood vessels (arrows). (H) and (I) FCD Illb: Albumin IR was detected in perivascular astrocytes (arrows). (J) and (K) TSC: Strong albumin IR was detected within the tuber in the perivascular region with expression in giant cells (arrows). Insert (a) in (J) shows albumin in cells surrounding a blood vessel (stained with CD31). Insert (b) in (J) and the insert in (K) show co-localization of albumin with GFAP within tubers. (L) For GG, there is strong albumin IR within the tumor. The insert shows co-localization of albumin with GFAP in tumor astrocytes. Scale bars (A): $40 \mu \mathrm{m} ;(\mathbf{B}): 25$ $\mu \mathrm{m} ;(\mathbf{C})-(\mathbf{E}): 20 \mu \mathrm{m} ;(\mathbf{F})-(\mathbf{H}),(\mathbf{J})$ and $(\mathbf{K})(\operatorname{bar}$ in $(\mathbf{J})): 80 \mu \mathrm{m} ;(\mathbf{I}),(\mathbf{L}): 40 \mu \mathrm{m}$. 
suggested that it plays a critical role in antigen-specific cytotoxicity [8]. MHC-I expression is necessary for antigen-specific cytotoxicity mediated by $\mathrm{CD} 8^{+}$lymphocytes [43]. In this context, we also detected $\mathrm{CD}^{+} \mathrm{T}$ lymphocytes with GrB in FCD IIB and TSC specimens in the vicinity of neurons. The possible contribution of a MHC-I restricted immune response to neuronal injury, occurring in patients with developmental pathologies and intractable epilepsy [18,44], requires further investigation.

MHC-I was also was found to be expressed in reactive microglial cells. Upregulation in microglial cells has been shown in both multiple sclerosis (MS) [45] and Rasmussen's encephalitis $[8,9]$. Interestingly, the microglial expression of MHC-I was significantly higher in FCD II compared to FCD I, reflecting the more prominent activation of microglial cells observed in FCD II specimens [6]. Moreover, MHC-I expression in microglia correlated positively with the duration of epilepsy, suggesting that the upregulation of MHC-I in these cells may also occur later in epileptogenesis.

Astrocytes did not display MHC-I immunoreactivity. Expression of MHC-I in astrocytes has also not been observed in MS lesions [45]. Thus upregulation of MHC-I in astrocytes appears to represent a specific feature of Rasmussen's encephalitis and an MHC-I restricted T-cell response has been suggested as critically contributing to the occurrence of the astrocytic degeneration observed in this pathology [9].

MHC-I expression was not detected in balloon cells in FCD IIb and in the large majority of giant cells in TSC. Interestingly, expression of MHC-I has been detected in the giant cells from different types of TSC-associated brain lesions in fetal cases ranging from 23 to 34 gestational weeks (GW) [46], indicating developmental changes in the phenotype of giant cells. MHC-I expression in these cells early in development may reflect their role as antigenpresenting cells and may account for the dynamic changes occurring early in development in TSC lesions.

We did not detect changes in the expression levels of MHC-I in endothelial cells within the different lesions examined. However, lesions (FCD IIB, TSC and GG) with prominent inflammatory changes and MHC-I upregulation in neurons and microglia displayed evidence of alterations in blood-brain barrier permeability, with albumin extravasation and uptake in astrocytes. These observations confirm previous findings for TSC [26], highlighting its similarity to FCD IIB and the differences with FCD I.

Our findings distinguish type I from type II FCD and indicate a prominent upregulation of MHC-I in neurons and microglial cells as part of the immune response occurring in epileptogenic glioneuronal lesions, such as FCD II, TSC and GG.

\section{Abbreviations}

AP: alkaline phosphatase; FCD: focal cortical dysplasia; GFAP: glial fibrillary acidic protein; GG: ganglioglioma; GrB: granzyme B; HLA: human leukocyte antigen; IgG: immunoglobulin G; IR: immunoreactivity; MHC: major histocompatibility complex; MIP: MHC-I peptide; MS: multiple sclerosis; NFKB: nuclear factor-kappa B; NeuN: neuronal nuclear protein; ODR: optical density ratio; SEM: standard error of mean; TSC: tuberous sclerosis complex.

\section{Competing interest}

None of the authors has any conflict of interest to disclose.

\section{Authors' contributions}

Immunohistochemistry and analysis of the data were performed by AP, Al and JA. EA helped with the analysis of the data. AI and AP helped EA in drafting and preparing the manuscript for submission. The overall experimental design was conceived and supervised by EA. WS and PR helped in the selection and collection of brain tissues. All authors read and approved the final manuscript.

\section{Acknowledgements}

This work was supported by the National Epilepsy Fund, 'Power of the Small', the Hersenstichting Nederland (NEF 09-05), EU FP7 project NeuroGlia (grant agreement number 202167) and KIKA (Stichting Kinderen Kankervrij; EA, AP). We confirm that we have read the Journal's position on issues involved in ethical publication and affirm that this report is consistent with those guidelines.

\section{Author details}

'Department of (Neuro)Pathology, Academic Medical Center, University of Amsterdam, Meibergdreef 9, Amsterdam 1105 AZ, The Netherlands. ${ }^{2}$ Departments of Pathology and Neurosurgery /Rudolf Magnus Institute for Neuroscience, University Medical Center Utrecht, Utrecht, The Netherlands. ${ }^{3}$ SEIN - Stichting Epilepsie Instellingen Nederland, Heemstede, The Netherlands. ${ }^{4}$ Swammerdam Institute for Life Sciences, Center for Neuroscience, University of Amsterdam, Amsterdam, The Netherlands.

Received: 14 November 2012 Accepted: 10 January 2013

Published: 24 January 2013

\section{References}


Epilepsia 2011, 52(Suppl 3):26-32.

2. Vezzani A, French J, Bartfai T, Baram TZ: The role of inflammation in epilepsy. Nature Reviews Neurology 2011, 7:31-40.

3. Vezzani A, Auvin S, Ravizza T, Aronica E: Glia-neuronal interactions in ictogenesis and epileptogenesis: role of inflammatory mediators. In Jasper's Basic Mechanisms of the Epilepsies. 4th edition. Edited by Noebels $\mathrm{J}$, Avoli M, Rogawski MA, Olsen RW, Delgado-Escueta AV. Bethesda, MD: 2012.

4. Aronica E, Ravizza T, Zurolo E, Vezzani A: Astrocyte immune responses in epilepsy. Glia 2012, 60:1258-1268.

5. Blumcke I, Thom M, Aronica E, Armstrong DD, Vinters HV, Palmini A, Jacques TS, Avanzini G, Barkovich AJ, Battaglia G, et al: The clinicopathologic spectrum of focal cortical dysplasias: a consensus classification proposed by an ad hoc Task Force of the ILAE Diagnostic Methods Commission. Epilepsia 2011, 52:158-174.

6. Iyer A, Zurolo E, Spliet WGM, van Rijen PC, Baayen JC, Gorter JA, Aronica E: Evaluation of the innate and adaptive immunity in type I and type II focal cortical dysplasias. Epilepsia 2010, 51(9):1763-1773.

7. Boulanger LM: MHC class I in activity-dependent structural and functional plasticity. Neuron Glia biology 2004, 1:283-289.

8. Bien CG, Bauer J, Deckwerth TL, Wiendl H, Deckert M, Wiestler OD, Schramm J, Elger CE, Lassmann H: Destruction of neurons by cytotoxic T cells: a new pathogenic mechanism in Rasmussen's encephalitis. Ann Neurol 2002, 51:311-318.

9. Bauer J, Elger CE, Hans VH, Schramm J, Urbach H, Lassmann H, Bien CG: Astrocytes are a specific immunological target in Rasmussen's encephalitis. Ann Neurol 2007, 62:67-80.

10. Engel JJ: Outcome with respect to epileptic seizures. In Surgical Treatment of the Epilepsies. Edited by Engel JJ. New York: Raven Press; 1993:609-621.

11. Gomez M, Sampson J, Whittemore V: The Tuberous Sclerosis Complex. Oxford: Oxford University Press; 1999 
12. Louis DN, Ohgaki H, Wiestler OD, Cavanee WK: WHO Classification of Tumours of the Central Nervous System. Lyon: IARC; 2007.

13. van Thiel PP, de Bie RM, Eftimov F, Tepaske R, Zaaijer HL, van Doornum GJ, Schutten M, Osterhaus AD, Majoie CB, Aronica E, et al: Fatal human rabies due to Duvenhage virus from a bat in Kenya: failure of treatment with coma-induction, ketamine, and antiviral drugs. PLoS Neglected Tropical Diseases [electronic resource] 2009, 3:428.

14. Ravizza T, Boer K, Redeker S, Spliet WG, van Rijen PC, Troost D, Vezzani A, Aronica E: The IL-1beta system in epilepsy-associated malformations of cortical development. Neurobiol Dis 2006, 24:128-143.

15. Aronica E, Gorter JA, Redeker S, Ramkema M, Spliet WG, van Rijen PC, Leenstra S, Troost D: Distribution, characterization and clinical significance of microglia in glioneuronal tumours from patients with chronic intractable epilepsy. Neuropathol App/ Neurobiol 2005, 31:280-291.

16. van der Loos CM: Multiple immunoenzyme staining: methods and visualizations for the observation with spectral imaging. J Histochem Cytochem 2008, 56:313-328.

17. Boer K, Troost D, Timmermans W, Gorter JA, Spliet WG, Nellist M, Jansen F, Aronica E: Cellular localization of metabotropic glutamate receptors in cortical tubers and subependymal giant cell tumors of tuberous sclerosis complex. Neuroscience 2008, 156:203-215.

18. Maldonado M, Baybis M, Newman D, Kolson DL, Chen W, McKhann G 2nd, Gutmann DH, Crino PB: Expression of ICAM-1, TNF-alpha, NF kappa B, and MAP kinase in tubers of the tuberous sclerosis complex. Neurobiol Dis 2003, 14:279-290.

19. Aronica E, Gorter JA, Redeker S, van Vliet EA, Ramkema M, Scheffer GL, Scheper RJ, van der Valk P, Leenstra S, Baayen JC, et al: Localization of breast cancer resistance protein (BCRP) in microvessel endothelium of human control and epileptic brain. Epilepsia 2005, 46:849-857.

20. Airas L, Lindsberg PJ, Karjalainen-Lindsberg ML, Mononen I, Kotisaari K, Smith DJ, Jalkanen S: Vascular adhesion protein-1 in human ischaemic stroke. Neuropathol Appl Neurobiol 2008, 34:394-402

21. Mizuguchi M, Takashima S: Neuropathology of tuberous sclerosis. Brain Dev 2001, 23:508-515.

22. DiMario FJ Jr: Brain abnormalities in tuberous sclerosis complex. J Child Neurol 2004, 19:650-657.

23. Baybis M, Yu J, Lee A, Golden JA, Weiner H, McKhann G 2nd, Aronica E, Crino PB: mTOR cascade activation distinguishes tubers from focal cortical dysplasia. Ann Neurol 2004, 56:478-487.

24. Schick V, Majores M, Engels G, Hartmann W, Elger CE, Schramm J, Schoch S, Becker AJ: Differential Pi3K-pathway activation in cortical tubers and focal cortical dysplasias with balloon cells. Brain Pathology 2007, 17:165-173.

25. Boer K, Troost D, Timmerman W, Spliet WGM, van Rijen PC, Aronica E: Pi3K-mTOR signaling and AMOG expression in epilepsy-associated glioneuronal tumors. Brain Pathology 2010, 20:234-244.

26. Boer $K$, Jansen F, Nellist M, Redeker S, van den Ouweland AM, Spliet WG van Nieuwenhuizen O, Troost D, Crino PB, Aronica E: Inflammatory processes in cortical tubers and subependymal giant cell tumors of tuberous sclerosis complex. Epilepsy Res 2008, 78:7-21.

27. Aronica E, Boer K, Becker A, Redeker S, Spliet WG, van Rijen PC, Wittink F, Breit T, Wadman WJ, Lopes da Silva FH, et al: Gene expression profile analysis of epilepsy-associated gangliogliomas. Neuroscience 2008, 151:272-292.

28. Maehlen J, Schroder HD, Klareskog L, Olsson T, Kristensson K: Axotomy induces MHC class I antigen expression on rat nerve cells. Neurosci Lett 1988, 92:8-13.

29. Pereira RA, Tscharke DC, Simmons A: Upregulation of class I major histocompatibility complex gene expression in primary sensory neurons, satellite cells, and Schwann cells of mice in response to acute but not latent herpes simplex virus infection in vivo. J Exp Med 1994, 180:841-850.

30. Neumann $\mathrm{H}$, Cavalie $\mathrm{A}$, Jenne DE, Wekerle $\mathrm{H}$ : Induction of MHC class I genes in neurons. Science 1995, 269:549-552.

31. Neumann $\mathrm{H}$, Schmidt $\mathrm{H}$, Cavalie A, Jenne D, Wekerle H: Major histocompatibility complex (MHC) class I gene expression in single neurons of the central nervous system: differential regulation by interferon (IFN)-gamma and tumor necrosis factor (TNF)-alpha. J Exp Med 1997, 185:305-316.

32. Kimura T, Griffin DE: The role of CD8(+) T cells and major histocompatibility complex class I expression in the central nervous system of mice infected with neurovirulent Sindbis virus. J Virol 2000, 74:6117-6125.
33. Foster JA, Quan N, Stern EL, Kristensson K, Herkenham M: Induced neuronal expression of class I major histocompatibility complex mRNA in acute and chronic inflammation models. J Neuroimmunol 2002, 131:83-91.

34. Neumann H: Control of glial immune function by neurons. Glia 2001 36:191-199

35. Neumann $\mathrm{H}$ : The immunological microenvironment in the CNS: implications on neuronal cell death and survival. J Neural Transm Suppl 2000, 59:59-68.

36. Boer K, Crino PB, Gorter JA, Nellist M, Jansen FE, Spliet WG, van Rijen PC, Wittink F, Breit T, Troost D, et al: Gene expression analysis of tuberous sclerosis complex cortical tubers reveals increased expression of adhesion and inflammatory factors. Brain Pathol 2010, 20:704-719.

37. Oeckinghaus A, Hayden MS, Ghosh S: Crosstalk in NF-kappaB signaling pathways. Nat Immunol 2011, 12:695-708.

38. Kesson AM, Cheng Y, King NJ: Regulation of immune recognition molecules by flavivirus, West Nile. Viral immunology 2002, 15:273-283.

39. Forloni M, Albini S, Limongi MZ, Cifaldi L, Boldrini R, Nicotra MR, Giannini G, Natali PG, Giacomini P, Fruci D: NF-kappaB, and not MYCN, regulates MHC class I and endoplasmic reticulum aminopeptidases in human neuroblastoma cells. Cancer Res 2010, 70:916-924.

40. Schild H, Rotzschke O, Kalbacher H, Rammensee HG: Limit of T cell tolerance to self proteins by peptide presentation. Science 1990, 247:1587-1589.

41. Caron E, Vincent K, Fortier MH, Laverdure JP, Bramoulle A, Hardy MP, Voisin G, Roux PP, Lemieux S, Thibault P, Perreault C: The MHC I immunopeptidome conveys to the cell surface an integrative view of cellular regulation. Mol Syst Biol 2011, 7:533.

42. Chen J, Tsai V, Parker WE, Aronica E, Baybis M, Crino PB: Detection of human papillomavirus in human focal cortical dysplasia type IIB. Ann Neurology 2012, 72:881-892.

43. Christinck ER, Luscher MA, Barber BH, Williams DB: Peptide binding to class I MHC on living cells and quantitation of complexes required for CTL lysis. Nature 1991, 352:67-70

44. Choi J, Nordli DR Jr, Alden TD, DiPatri A Jr, Laux L, Kelley K, Rosenow J, Schuele SU, Rajaram V, Koh S: Cellular injury and neuroinflammation in children with chronic intractable epilepsy. J Neuroinflammation 2009, 6:38.

45. Gobin SJ, Montagne L, Van Zutphen M, Van Der Valk P, Van Den Elsen PJ, De Groot CJ: Upregulation of transcription factors controlling MHC expression in multiple sclerosis lesions. Glia 2001, 36:68-77.

46. Prabowo AS, Anink JJ, Lammens M, Nellist M, van den Ouweland AM, Adle-Biassette $H$, Sarnat HB, Flores-Sarnat L, Crino PB, Aronica E: Fetal brain lesions in tuberous sclerosis complex: TORC1 activation and inflammation. Brain Pathology 2012, 23:45-59.

\section{doi:10.1186/1742-2094-10-12}

Cite this article as: Prabowo et al.: Differential expression of major histocompatibility complex class I in developmental glioneuronal lesions. Journal of Neuroinflammation 2013 10:12.

\section{Submit your next manuscript to BioMed Central and take full advantage of:}

- Convenient online submission

- Thorough peer review

- No space constraints or color figure charges

- Immediate publication on acceptance

- Inclusion in PubMed, CAS, Scopus and Google Scholar

- Research which is freely available for redistribution 